\section{Cooling Accelerates Flowering of Lysimachia clethroides Duby}

\author{
Pamela M. Lewis ${ }^{1}$, Allan M. Armitage ${ }^{2}$, and James M. Garner ${ }^{3}$ \\ Department of Horticulture, The University of Georgia, Athens, GA 30602-7273
}

Additional index words. Gooseneck loosestrife, case cooling, stem length, stem diameter

Abstract. The effect of cooling method and duration on off-season cut flower production of Lysimachia clethroides Duby was examined. Rhizomes harvested in October were cooled for $0,4,6,8,10$, or 12 weeks at $4 \pm 1^{\circ} \mathrm{C}$ in crates with unmilled sphagnum peat moss or in 3.75-L pots filled with a commercial soilless medium prior to forcing in a warm greenhouse. After 6 or more weeks of cooling, shoots emerged from crates in higher percentages than from pots. However, only the duration of cooling, not the method, affected the rate of shoot emergence, visible bud formation, and anthesis of the first bud in the raceme. As cooling increased from 0 to 12 weeks, the greenhouse days required for shoot emergence, visible bud formation, and anthesis decreased linearly. The number of flowering flushes and flowering stems produced per plant varied quadratically with cooling duration, and the highest yields occurred when rhizomes received between 4 and 10 weeks of cooling. High numbers of flowers were produced rapidly after 10 weeks of cooling. As the number of successive flowering flushes increased, the stem length increased linearly while the stem diameter decreased linearly.

Lysimachia clethroides Duby, gooseneck loosestrife, is a herbaceous perennial native to temperate regions of China and Japan (Bailey, 1924). Although generally grown as an ornamental landscape plant, elegant white racemes and long stems make this plant well suited for specialty cut flower production (Armitage, 1993). Under field cultivation, cut flowers of L. clethroides may be harvested for several weeks during the summer. However, the yearround demand for specialty cut flowers has led to increased interest in off-season greenhouse production of the species. Temperate perennials such as L. clethroides often flower only after exposure to specific environmental conditions, and growers must be aware of these requirements to successfully force flowers in the greenhouse (Thomas, 1993).

Vernalization, the induction or promotion of flowering by low temperatures, is common in both perennial and biennial species (Bernier et al., 1981; Thomas, 1993). A vernalization requirement may be qualitative or quantitative, and may or may not be accompanied by a photoperiod requirement. In plants with a quantitative vernalization response, cooling may not be required for flowering but flowering is enhanced by exposure to a period of low temperatures. For most perennials, e.g., Eustoma russellianum (Hook.) G. Don ex Sweet and Campanula pyramidalis L. (Pergola, 1992; Zimmer, 1983), cooling at 1 to 7

Received for publication 15 Jan. 1998. Accepted for publication 8 Sept. 1998. We thank Here \& Now Gardens, Gales Creek, Ore., for donating the rhizomes used in this study. The cost of publishing this paper was defrayed in part by the payment of page charges. Under postal regulations, this paper therefore must be hereby marked advertisement solely to indicate this fact.

${ }^{1}$ Research Specialist.

${ }^{2}$ Professor of Horticulture.

${ }^{3}$ Graduate Research Assistant.
${ }^{\circ} \mathrm{C}$ for 4 to 12 weeks is sufficient to fulfill cooling requirements, with the promotion of flowering typically becoming stronger as the cooling period increases and the cooling temperature decreases. In some species, flower quality may also be affected by cold exposure. Cooled corms of Freesia $\times$ hybrida L.H. Bailey flower earlier and have shorter scapes, leaves, and spikes than do their noncooled counterparts (Wulster and Gianfagna, 1991).

Iversen and Weiler (1994) investigated methods to force flowering of $L$. clethroides and other garden perennials for winter flower shows. They found that L. clethroides is a long-day plant that emerges in higher percentages and flowers more rapidly as low-temperature exposure increases from 0 to 12 weeks. Iversen and Weiler used rhizomes individually potted in $15-\mathrm{cm}$ containers, but cooling potted rhizomes may not be practical for cut flower production. Most producers force cut flowers directly in greenhouse beds, not in individual containers, and case cooling of $L$. clethroides rhizomes may be more practical. The objectives of this study were to: 1) determine if case cooling is as effective as pot cooling, 2) identify the minimum cooling duration required to produce high emergence percentages and rapid flowering, and 3) evaluate the effects of cooling on quality and yield of cut flower stems.

\section{Materials and Methods}

On 10 Oct. 1995, 500 L. clethroides rhizomes were harvested from outdoor production beds (Here \& Now Garden, Gales Creek, Ore.), packed into plastic bags with milled sphagnum peat moss, and shipped to Athens, $\mathrm{Ga}$. Upon arrival, the rhizomes were sorted and 240 uniform rhizomes were selected. One hundred of these rhizomes were placed in plastic crates $(36 \times 34 \times 27 \mathrm{~cm})$ lined with clear, 0.2-mm (8-mil) polyethylene. Inside the crates, rhizomes were arranged between layers of moist, unmilled sphagnum peat moss. No two rhizomes in a layer overlapped, and each crate contained five layers of rhizomes.

The remaining 140 rhizomes were potted in black, 3.8-L containers filled with a commercial soilless medium (Fafard 3-B, Conrad Fafard Inc., Anderson, S.C.) and thoroughly watered. Forty randomly selected pots were transferred to a glass greenhouse and the remaining pots and crates were stored in darkness in a cooler at $4 \pm 1^{\circ} \mathrm{C}$. To allow aeration, the tops of the crates and pots were left uncovered during cooling. At the commencement of cooling treatments, the unmilled sphagnum peat moss contained $\approx 90 \%$ water by weight, while the soilless medium contained $\approx 74 \%$. Tap water was added as needed during cooling to maintain uniform moisture within the pots and crates.

After 4, 6, 8, 10, and 12 weeks of cooling, 20 randomly selected pots were removed from the cooler. Also at this time, 20 rhizomes were removed from the crates and potted into $3.8-\mathrm{L}$ containers filled with Fafard Mix No. 3-B. An equal number of rhizomes were selected from each layer of every crate. The selected pot- and crate-cooled rhizomes were then transferred to the glass greenhouse and placed on benches in a completely randomized design.

Pots were irrigated with tap water until shoots began to emerge. After emergence, plants received $200 \mathrm{mg} \cdot \mathrm{L}^{-1} \mathrm{~N}$ by alternating $15 \mathrm{~N}-9.9 \mathrm{P}-14.1 \mathrm{~K}$ and $15 \mathrm{~N}-0 \mathrm{P}-12.5 \mathrm{~K}$ (Peter's 15-16-17 Peat-lite Special and 15-0-15 Dark Weather Feed, respectively; The Scotts Co., Marysville, Ohio) at each irrigation. To prevent accumulation of excessive soluble salts, the pots were leached once a week with tap water. Plants received night interruption lighting from 2200 to $0200 \mathrm{HR}$ with 40 -W incandescent lamps (Sylvania, St. Marys, Pa.) providing an average intensity of $14 \mu \mathrm{mol} \cdot \mathrm{m}^{-2} \cdot \mathrm{s}^{-1}$ at plant level. Greenhouse temperatures were maintained at $20 \pm 5{ }^{\circ} \mathrm{C}$ day $/ 19 \pm 3{ }^{\circ} \mathrm{C}$ night.

The number of days until shoot emergence, first visible bud formation, and anthesis was recorded for each plant in every treatment. A raceme was considered to have attained anthesis when the first flower of that raceme was completely open. Stems were harvested when about one-third of all the flowers in a raceme were open $-\approx 1 \mathrm{~d}$ after anthesis of the first flower in the inflorescence. If the raceme was produced on a side branch of the main shoot, the stem was cut at the junction of the branch and the main shoot. Stems with terminal racemes on main shoots were cut about four nodes $(\approx 10 \mathrm{~cm})$ above the crown of the plant. Data collected from the flowering stems were length and width of individual racemes, stem length and diameter, stem yield per rhizome, and number of flowering flushes per rhizome. Raceme length was measured from the axil of the lowest flower in the raceme to the tip of the uppermost bud, and width was measured at the middle of the raceme. Stem length was measured from the cut end of the stem to the axil of the lowest flower, and stem diameter was measured with calipers at the base of the 
swelling of the lowest node on the cut flower stem. A flowering flush was defined as a group of racemes that attained anthesis within a period of $10 \mathrm{~d}$. All data were tested using the SAS General Linear Model procedure (SAS Institute, Cary, N.C.). Variation within cooling duration was determined by orthogonal contrasts, and cooling method means were separated by Tukey's (HSD) studentized range test.

\section{Results and Discussion}

Cooling method (pot cooling vs. crate cooling) strongly influenced shoot emergence. With 4 weeks of cooling, $75 \%$ of both pot- and crate-cooled rhizomes produced shoots, but when cooling continued beyond 4 weeks, cratecooled rhizomes emerged in much higher percentages ( $75 \%$ to $95 \%$ ) than did pot-cooled rhizomes (40\% to $75 \%$; Fig. 1). Differences in soluble salts concentrations and $\mathrm{pH}$ between the amended commercial medium and the unmilled sphagnum moss crates may account for differences in shoot emergence between pot- and crate-cooled rhizomes.

Shoot emergence of both pot- and cratecooled rhizomes was $<100 \%$ after 12 weeks of cooling in our study, while Iversen and Weiler (1994) reported that shoots emerged from all plants after 12 weeks of cooling. We used dormant, bare-root rhizomes, while Iversen and Weiler used rhizomes with leafy shoots that were divided and potted in August and grown at $15.5^{\circ} \mathrm{C}$ nights under 8 -h photoperiods for 2 months before cutting back the shoots and initiating experimental treatments. Plants used in their study probably had more developed root systems with higher carbohydrate concentrations when cooling began, compared with those used in our study.

Although cooling method influenced the number of plants emerging, only cooling duration affected the rate of shoot emergence and flowering. Therefore, data on the rate of emergence and flowering for crate- and pot-cooled rhizomes were combined. As cooling increased from 0 to 12 weeks, the number of greenhouse days required for shoot emergence, visible bud formation, and anthesis of the first raceme decreased in a linear manner (Fig. 2).

Stems from uncooled rhizomes of $L$. clethroides emerged and flowered, although at slower rates than cooled rhizomes. Cooling accelerated flowering by decreasing the time required for the production of visible buds. The number of days between emergence and visible bud formation decreased as cooling duration increased, but the number of days between visible bud formation and anthesis of the first flower differed by $<5 \mathrm{~d}$ among all treatments. Therefore, although cooling accelerated emergence and reproductive development in L. clethroides, it was not required for induction of flowering.

The trend for number of flowering stems per plant was quadratic within cooling duration (Fig. 3a). Rhizomes exposed to 4, 6, 8, or 10 weeks of cooling produced an average of three or more stems per plant, while uncooled rhizomes and those cooled for 12 weeks produced about half as many. Iversen and Weiler

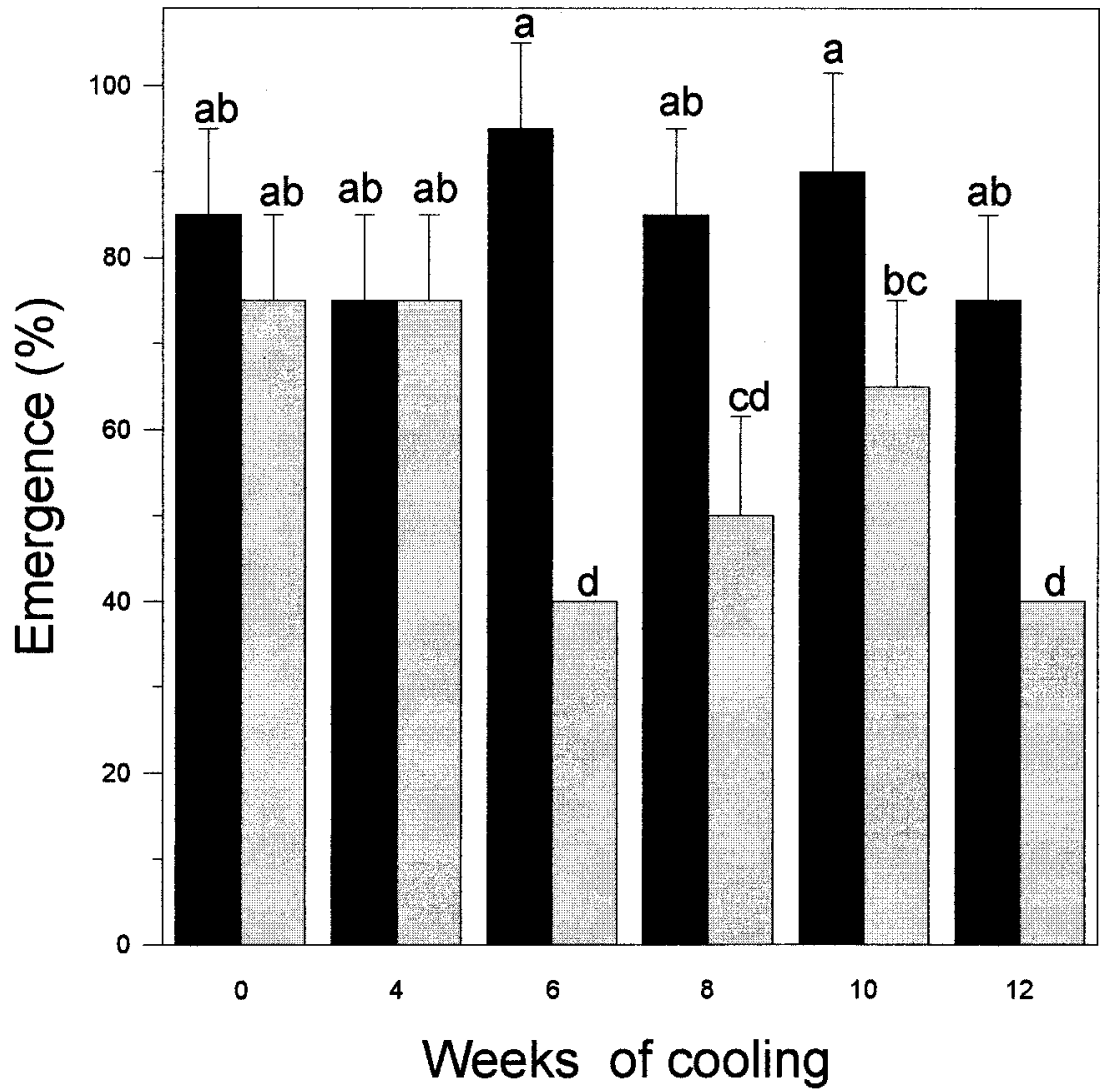

Fig. 1. The influence of cooling method and duration on shoot emergence of Lysimachia clethroides. Mean separation by Tukey's Studentized range (HSD) test at $P \leq 0.05$.

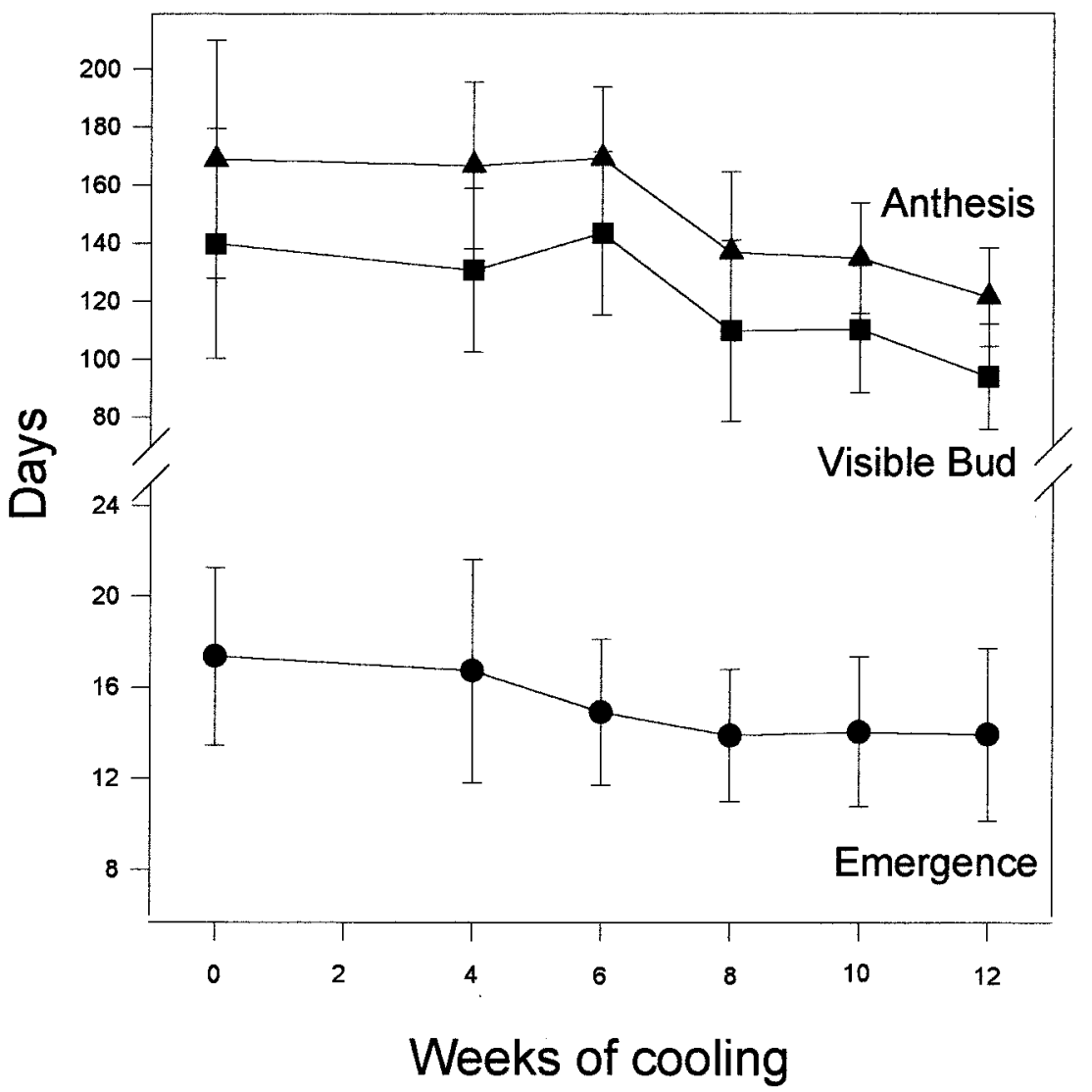

Fig. 2. The influence of cooling duration on the number of greenhouse days required for shoot emergence, visible bud formation, and anthesis for Lysimachia clethroides. Means are for combined cooling methods. All trends are linear, $P \leq 0.001$. 
(1994) reported that cooling increased flowering stem counts for plants with established root systems, with the highest number of stems produced after 12 weeks of cooling. In our study, stem yield declined after 10 weeks of cooling, possibly because of a lower carbohydrate reserve status in the bare-root rhizomes we used. The trend for number of flowering flushes per plant was also quadratic within cooling duration, with the highest number of flushes occurring in plants receiving between 4 and 10 weeks of cooling. Racemes were longest when 8 weeks of cooling were provided and shortest when no cooling was given, exhibiting a quadratic trend within cooling duration (Fig. 3b). Raceme width was similar among all treatments (data not shown).

Stem lengths and diameters for $L$.
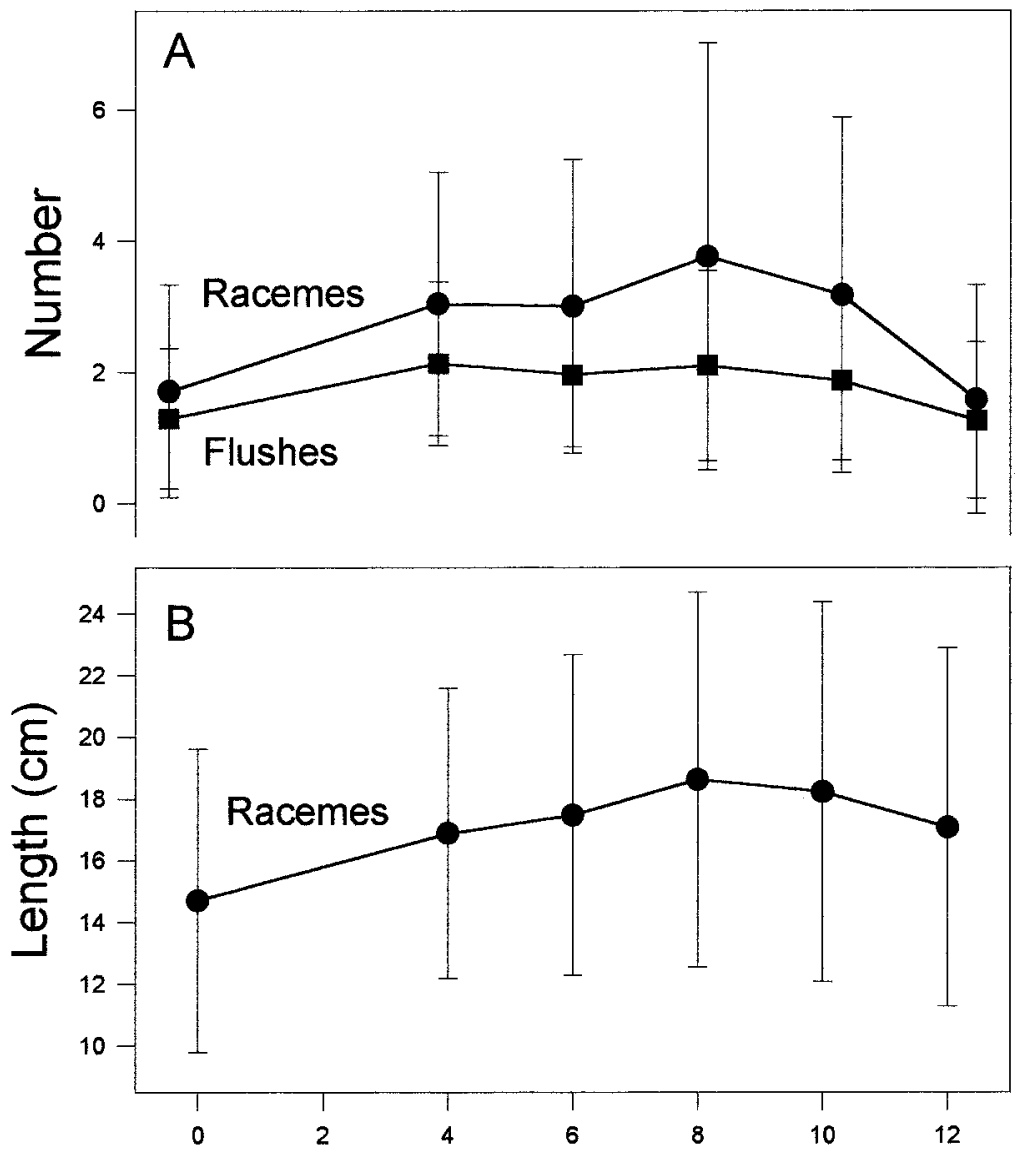

Weeks of cooling

Fig. 3. The influence of cooling duration on (A) numbers of racemes and flushes, and (B) raceme length of Lysimachia clethroides. Values represent means for combined cooling methods. All trends are quadratic at $P \leq 0.001$ clethroides in crates and pots were similar among the six cooling durations. Racemes produced during the first flush of flowering had the shortest stem lengths and the largest stem diameters compared with those produced in later flushes. Increase in stem length was generally linear with each successive flush, regardless of cooling technique (Table 1), while stem diameter decreased linearly with each successive flush.

Cut flower production of $L$. clethroides was enhanced by cooling, suggesting a quantitative vernalization response. Case cooling of dormant, bare-root rhizomes in moist, unmilled sphagnum moss was more effective than pot cooling in an amended commercial medium. Shoot emergence and flower development accelerated as cooling duration increased from 0 to 12 weeks, and highest cut flower production occurred in rhizomes cooled for 4 to 10 weeks. Therefore, 10 weeks of cooling prior to greenhouse forcing appears to maximize cut flower yield and raceme quality.

\section{Literature Cited}

Armitage, A.M. 1993. Specialty cut flowers. Varsity Press/Timber Press, Portland, Ore.

Bailey, L.H. 1924. Manual of cultivated plants. Macmillan, New York.

Bernier, G., J.M. Kinet, and R.M. Sachs. 1981. The physiology of flowering. Vol. 1. Control by low temperature. CRC Press, Boca Raton, Fla.

Iversen, R.R. and T.C. Weiler. 1994. Strategies to force flowering of six herbaceous garden perennials. HortTechnology 4:61-65.

Pergola, G. 1992. The need for vernalization in Eustoma russellianum. Scientia Hort. 51:123127.

Thomas, B. 1993. Internal and external controls on flowering, p. 1-19. In: B.R. Jordan (ed.). The molecular biology of flowering. CAB International, Wallingford, U.K.

Wulster, G.J. and T.J. Gianfagna. 1991. Freesia hybrida respond to ancymidol, cold storage of corms, and greenhouse temperatures. HortScience 26:1276-1278.

Zimmer, K. 1983. Campanula pyramidalis Studies IV. Low temperature requirement (in German). Gartenbauwissenschaft 48:23-27.

Table 1. The influence of flush, temperature, and cooling method on stem length and diameter of Lysimachia clethroides cooled for $0,4,6,8,10$, or 12 weeks in crates vs. pots.

\begin{tabular}{|c|c|c|c|c|c|c|c|c|c|c|c|c|}
\hline \multirow[b]{3}{*}{ Flush } & \multicolumn{12}{|c|}{ Weeks of cooling } \\
\hline & \multicolumn{2}{|c|}{0} & \multicolumn{2}{|c|}{4} & \multicolumn{2}{|c|}{6} & \multicolumn{2}{|c|}{8} & \multicolumn{2}{|c|}{10} & \multicolumn{2}{|c|}{12} \\
\hline & Crate & Pot & Crate & Pot & Crate & Pot & Crate & Pot & Crate & Pot & Crate & Pot \\
\hline \multicolumn{13}{|c|}{ Stem length $(\mathrm{cm})$} \\
\hline Second & 87.3 & 87.5 & 69.3 & 79.3 & 86.6 & 90.8 & 76.4 & 77.2 & 76.7 & 100.1 & 71.6 & 68.0 \\
\hline Third & 96.0 & 92.8 & 74.1 & 99.0 & 88.3 & 102.4 & 81.5 & 94.7 & 92.0 & 118.5 & 83.1 & 81.7 \\
\hline $\mathrm{L}^{\mathrm{z}}$ & $* *$ & $* *$ & NS & $* * *$ & $* *$ & $* * *$ & $* *$ & $* * *$ & $* * *$ & *** & $*$ & $*$ \\
\hline Second & 3.85 & 4.03 & 4.31 & 4.37 & 4.38 & 4.14 & 4.47 & 4.23 & 4.52 & 4.34 & 5.01 & 4.76 \\
\hline Third & 3.93 & 3.67 & 3.83 & 3.84 & 4.19 & 3.91 & 3.80 & 3.64 & 4.00 & 4.02 & 3.77 & 3.91 \\
\hline $\mathrm{L}$ & $* * *$ & $* *$ & NS & $* * *$ & $*$ & $* * *$ & $* * *$ & $* * *$ & $* * *$ & NS & $* * *$ & $*$ \\
\hline Q & $* *$ & NS & NS & NS & NS & NS & NS & $* *$ & NS & NS & NS & NS \\
\hline
\end{tabular}

${ }^{\mathrm{Q}} \mathrm{L}, \mathrm{Q}$ : linear or quadratic response.

Ns, ***,****Nonsignificant, or significant at $P \leq 0.05,0.01$, or 0.001 , respectively. 